\title{
Widespread mitovirus sequences in plant genomes
}

Jeremy A Bruenn, Benjamin E. Warner, Pradeep Yerramsetty

The exploration of the evolution of RNA viruses has been aided recently by the discovery of copies of fragments or complete genomes of non-retroviral RNA viruses (Non-retroviral Endogenous RNA Viral Elements, or NERVEs) in many eukaryotic nuclear genomes. Among the most prominent NERVEs are partial copies of the RNA dependent RNA polymerase (RdRP) of the mitoviruses in plant mitochondrial genomes. Mitoviruses are in the family Narnaviridae, which are the simplest viruses, encoding only a single protein (the RdRP) in their unencapsidated viral plus strand. Narnaviruses are known only in fungi, and the origin of plant mitochondrial mitovirus NERVEs appears to be horizontal transfer from plant pathogenic fungi. At least one mitochondrial mitovirus NERVE, but not its nuclear copy, is expressed. 
2 Widespread mitovirus sequences in plant genomes

3

Jeremy A. Bruenn, Benjamin E. Warner, and Pradeep Yerramsetty

Department of Biological Sciences

State University of New York at Buffalo

Buffalo, NY 14260

*Corresponding author

J.A. Bruenn

cambruen@buffalo.edu

B.E. Warner

bewarner@buffalo.edu

P. Yerramsetty

pradeepk@buffalo.edu 


\section{INTRODUCTION}

The Narnaviridae is one of three known RNA virus families in which the viral genome is not encapsidated in a protein coat. The Narnaviridae (Esteban et al. 1994; Esteban et al. 1992; Garcia-Cuellar et al. 1995; Hillman \& Cai 2013; Hong et al. 1998; Osaki et al. 2005; RodriguezCousino et al. 1991; Rodriguez-Cousino \& Esteban 1992) and the Hypoviridae (Koonin et al. 1991) are presently known exclusively in fungi and the Endoviridae in plants (Fukuhara et al. 2006; Gibbs et al. 2000). All are related to families of encapsidated RNA viruses and have been proposed to be derived from common ancestors via loss of capsid polypeptide genes (Koonin \& Dolja 2013; Roossinck 2010). As judged by the sequences of their RNA-dependent RNA polymerases (RdRPs), the closest relatives of the Narnaviridae are the leviviruses of bacteria (Garcia-Cuellar et al. 1997) and the ourmiaviruses of plants (Rastgou et al. 2009). All of the known Narnaviridae of plant pathogenic fungi are mitoviruses: unencapsidated plus strand RNA viruses infecting mitochondria (Hillman \& Cai 2013). There may be Narnaviridae of other organisms, but possible contamination with fungi has so far prevented their identification in insects (Cook et al. 2013), although they have been identified in oomycetes (Cai et al. 2012), which have similarities to both fungi and plants. Narnaviridae have not been identified in plants (Al Rwahnih et al. 2011). The association of Narnaviridae with mitochondria is suggestive, given that their closest relatives, the leviviruses, are viruses of bacteria and the origin of mitochondria is from bacterial endosymbionts (Raven 1970). However, rather than populating the mitochondria of all eukaryotes, the Narnaviridae have only been found in fungi. The Narnaviridae are the simplest viruses, with a single RNA segment encoding a single protein, the RdRP.

The exploration of the evolution of RNA viruses has been aided recently by the discovery of copies of fragments or complete genomes of non-retroviral RNA viruses (Non-retroviral Endogenous RNA Viral Elements, or NERVEs) in many eukaryotic nuclear genomes. (Ballinger et al. 2013; Ballinger et al. 2012; Crochu et al. 2004; Cui \& Holmes 2012; Horie et al. 2010; Katzourakis \& Gifford 2010; Taylor \& Bruenn 2009; Taylor et al. 2011; Taylor et al. 2010). The Narnaviridae do have paleovirus sequences in eukaryotic genomes. However, these are not in fungal genomes, where they might be expected, but in plant genomes (Hong et al. 1998; Marienfeld et al. 1997). These mitovirus NERVEs are prominent in plant mitochondria, where they cannot be mistaken for contaminating sequences, because they are present in complete, relatively small, circular genomes. We examined a large collection of these mitovirus NERVEs to explore their origin, which has been ambiguous (Shackelton \& Holmes 2008). It has been suggested that the mitoviruses arose from plant genetic elements (Shackelton \& Holmes 2008). However, our results suggest the opposite - one or more integration events of a fungal mitovirus cDNA in the mitochondrial DNA of vascular plants.

\section{MATERIALS AND METHODS}

\section{Bioinformatics}

Predicted amino acid sequences of NERVEs were obtained using the BLAST (Altschul et al. 1990; Altschul et al. 1997) tblastn algorithm with a number of mitovirus RdRp query sequences and the NCBI (National Center for Biotechnology Information, Bethesda, USA) Viridiplantae subset of the refseq DNA sequence database. NERVEs were identified as having significant $\mathrm{E}$ values (usually less than $10^{-5}$ ) and/or as preserving the known conserved motifs (Bruenn 2003) in the RdRp. 
We found 175 mitovirus NERVEs in the genomes of completely sequenced mitochondria. Sequences were aligned using MAFFT (Katoh et al. 2002) with the E-INS-I algorithm, a BLOSUM62 matrix, and a gap penalty of 1.53 implemented in Geneious (Kearse et al. 2012). Maximum likelihood phylogenetic analysis was carried out with PhyML 3.0 as implemented in Seaview 4.3.5 (Anisimova \& Gascuel 2006) using the model optimization of Prottest (Abascal et al. 2005), which prescribed the VT+invariable sites parameter (I) + gamma parameter for among-site variation (G)+ empirically-determined amino acid frequencies (F). Given that the Leviviridae are most closely related to the Narnaviridae (Shackelton \& Holmes 2008) (generally about 24\% identity, E value of 0.23 ), two representative levivirus sequences were used to root the tree. Successful alignment of all of the conserved motifs of the RdRp is indicative of an accurate alignment. When alignments were evaluated with TCS (Chang et al. 2014) and scores less than 8 filtered out (a very conservative choice), the position of the mitovirus NERVEs within the mitoviruses (Fig. 4) was unaltered. Synteny was assessed and visualized using the CoGe server (https://genomevolution.org/CoGe/) as described (Lyons \& Freeling 2008).

RNA and DNA isolation

To examine the possible functional significance of mitovirus NERVEs, the cellular expression of a representative mitovirus NERVE was examined in Arabidopsis. RNA (and DNA) was isolated from the leaves of 6 week old Arabidopsis thaliana plants using an RNeasy Plant Mini Kit (Qiagen) according to the manufacturer's protocol.

The concentration of extracted nucleic acids was measured using a Nanodrop spectrophotometer and the quality monitored by formaldehyde agarose gel electrophoresis. The final preparation (0.36 [g/0l) contained DNA as well as total RNA and was treated with DNase to measure transcripts. $15 \mu \mathrm{l}$ of the preparation was treated with $3 \mu \mathrm{l}$ of RQ1 RNase-free DNAse (1 unit/ $\mu \mathrm{g}$ RNA, Promega) in a $30 \mu \mathrm{l}$ reaction after the addition of $3 \mu \mathrm{l}$ of RQ11 RNase-free DNase 10x reaction buffer and $9 \mu \mathrm{l}$ double-distilled water for 30 minutes at $37^{\circ} \mathrm{C}$. The reaction was stopped with $3 \mu \mathrm{l}$ DNase stop solution (20mM EGTA) and incubated at $65^{\circ} \mathrm{C}$ for 10 minutes to denature the DNase prior to RTPCR.

PCR and RTPCR

The synthetic DNA oligonucleotides used for PCR and RTPCR of the Arabidopsis thaliana mitovirus NERVEs were CTTGCTCGCTTTGGCAGGAAG (5' sequence for chromosomal copy), GCGGCGTTTGTTTGTAATCGGT (5' sequence for the mitochondrial copy) and CAATGCACGATGCCATCGTTTGA ( 3 ' sequence for both copies). The oligonucleotides used for the control (rbcL) sequence were TCAGGT GGACGAAAGTGTAAAG and GAACCACTCCCAGTTAGCATAG.

Pre-incubation mixes consisted of $10 \mu \mathrm{l}$ of template ( $200 \mathrm{ng}$ ), $1 \mu \mathrm{l}$ of $10 \mathrm{mM}$ dNTPs, and $1.5 \mu \mathrm{l}$ of each primer $(10 \mu \mathrm{M})$. RTPCR reactions were $14 \mu \mathrm{l}$ of pre-incubation mix, $5 \mu \mathrm{l}$ of double distilled water, $5 \mu$ of $5 x$ Qiagen buffer, and $1 \mu$ l of Qiagen one-step RTPCR enzyme mix. DNA synthesis reactions consisted of one cycle at $50^{\circ} \mathrm{C}$ for 30 minutes; one cycle of $95^{\circ} \mathrm{C}$ for 10 minutes; 40 cycles of $94^{\circ} \mathrm{C}$ for 15 seconds, $55^{\circ} \mathrm{C}$ for 15 seconds, $72^{\circ} \mathrm{C}$ for 30 seconds and a final cycle of $72^{\circ} \mathrm{C}$ for 10 minutes followed by cooliing at $12^{\circ} \mathrm{C}$ for 10 minutes and refrigeration at $4^{\circ} \mathrm{C}$. Analysis of products was by electrophoresis in $1.4 \%$ agarose gels run with $1 \mu \mathrm{g} / \mathrm{ml}$ ethidium bromide in 1XTAE. 


\section{Mitovirus NERVEs in mitochondrial genomes}

The mitovirus NERVEs present in plants vary from nearly complete versions of the RdRP to remnants barely detectable in sequence searches ( $E$ values varying from $10^{-40}$ to 0.01 ). For simplification, we primarily limit our discussion to the mitovirus NERVEs present in completely sequenced mitochondrial genomes of plants, which currently derive from 90 different organisms. In these mitochondrial genomes, there are more than 175 partial or nearly complete copies of the mitovirus RdRP. In some cases, there is more than one mitovirus NERVE on a single mitochondrial DNA. In others, there is more than one mitochondrial genome per cell (e.g. Amborella), some of which have mitovirus NERVEs. Remarkably, an alignment of these 175 NERVEs shows that all the well-known conserved motifs (A-F) of the RdRP (Bruenn 2003) are preserved in the paleovirus copies (Fig. 1, Supplemental Figs. 1a and b). This is reminiscent of the perfect preservation of the totivirus conserved motifs in totivirus RdRP NERVEs (Taylor \& Bruenn 2009).

In many cases, there are mitovirus NERVEs both in mitochondrial and nuclear genomes and the direction of transfer is clear from an examination of the synteny of the miotochondrial and nuclear genomes in the region containing the mitovirus NERVE (Fig. 2), since genes of mitochondrial origin now appear in the nuclear genome. As is well known, blocks of mitochondrial DNA have been transferred to the nuclear genome (Leister \& Kleine 2011). In the cases shown, these blocks included mitovirus NERVEs. There are many mitovirus NERVEs in plant mitochondrial and/or nuclear genomes not included in our survey because the mitochondrial genomes of their plants have not been completely sequenced. For instance, there are at least 5 mitovirus NERVEs in the tomato genome, but its completed mitochondrial genome is not yet available. Consequently we cannot say that every mitovirus NERVE present in the plant nuclear genome is derived from a mitochondrial copy, but where comparisons can be made this appears to be the case.

The phylogeny of plant mitochondria does not accurately represent accepted plant phylogeny, probably because of frequent horizontal transfer of mitochondrial genes (Xi et al. 2013). There is also very poor resolution among closely related species, which is a consequence of the great degree of sequence conservation in coding regions but complete lack of synteny among plant mitochondrial genomes (Palmer \& Herbon 1988). Given the rate of scrambling of mitochondrial genomes, the existence of synteny between mitochondrial and nuclear genomes within blocks of transferred mitochondrial DNA (Fig. 2) implies recent transfer events.

The plant mitochondria containing mitovirus NERVEs are all seed plants (Spermatophyta) or club mosses (Lycopodiophyta). Mitovirus NERVEs are missing from all of the Chlorophyta and from all of the Streptophyta except for the Embryophyta (higher plants) and within the higher plants are confined to the Tracheophyta (vascular plants). Among the Viridiplantae, there are sequences for mitochondria from Chlorokybophyceae (Chlorokybus atmophyticus) , Mesostigmatophyceae (Mesostigma viride), Charophyceae (Chara vulgaris) , Coleochaetophyceae (Chaetosphaeridium globosum), Anthocerotophyta (Megaceros aenigmaticus), Bryophyta (Anomodon attenuates), and Marchantiophyta (Marchantia polymorpha) and all are lacking mitovirus NERVEs (Supplemental Table 1). Among the Tracheophyta (vascular plants), there are examples only from the Lycopodiophyta (Huperzia squarrosa) and Spermatophyta (all the other NERVE containing mitochondria) and mitovirus NERVEs are present in both. Our data are therefore consistent with an original integration of mitovirus cDNA in the mitochondria of the common ancestor of the vascular plants (Tracheophyta), as shown in the tree adapted from Davis 
et al (Davis et al. 2014), Fig. 3. However, we cannot exclude multiple integration events subsequent to the origin of vascular plants.

The plant mitochondrial mitovirus NERVEs are all derived from one or more fungal mitovirus RdRP genes, as shown in the phylogram of Fig. 4. The phylogram was derived from an alignment of the 61 plant mitovirus NERVEs longer than 100 amino acids with all of the known Narnaviridae (31 viruses including the two Saccharomyces cerevisiae viruses) and rooted by the leviviruses (alignment is shown as Supplemental Fig. 2). It places the mitovirus NERVEs clearly within the mitoviruses. All of the main branch support values are reasonable. Since all of the mitoviruses are present in plant pathogenic fungi, transfer of mitoviruses from their fungal hosts to plant mitochondria is not inconceivable.

There is a single prominent group of seed plants missing mitovirus NERVEs (the Poales, including Zea mays and Oryza sativa) (Supplementary table 1). Given the wide distribution of mitovirus NERVEs in the vascular plants, this is likely to have resulted from a loss rather than from multiple integration events. Actually, the vestiges of mitovirus NERVEs are still detectable in some of the grains, for instance in the Sorghum bicolor nuclear genome, with a region of chromosome 6 with similarity (E value 0.26 ) to the ABCDE portion of the mitovirus RdRP (Supplementary Fig. 3).

\section{Expression of mitovirus NERVES}

The widespread preservation of NERVEs (and their open reading frames) in eukaryotes suggests that they may have important functions. A prerequisite for function is expression. We chose to examine the expression of a single mitovirus NERVE in Arabidopsis whose mitochondrial and nuclear expression can be distinguished. Many of the mitovirus NERVEs preserve much or all of the RdRP amino acid sequence in long open reading frames. One of these is the NERVE in Arabidopsis, which has copies in both nuclear and mitochondrial genomes (Fig. 2). This is a region of 274 amino acids in the mitochondrial copy and 246 amino acids in the nuclear copy, both of which encompass the entire FABCDE conserved region of the RdRP and end at the same residue. Since these sequences are located at the extreme end of a syntenous region, it was possible to construct oligonucleotide primers in which one primer resides in the conserved RdRP region and the other in the unique, non-syntenous nuclear or mitochondrial DNA adjacent to the common region but possibly on the same transcriptional unit. Hence an RT-PCR experiment can determine if one or both of these sequences are transcribed. Total RNA from Arabidopsis thaliana (with some contaminating DNA) was subject to RT-PCR using these primers. The result (Fig. 5) shows that only the mitochondrial NERVE is transcribed. The conservation of the core RdRP sequence in a long open reading frame and its transcription are consistent with selection for expression of the NERVE protein, possibly as a means of interfering with mitovirus propagation, which, presumably, would occur in the mitochondrion. The mechanism of interfence might be at the protein level (Taylor et al. 2013; Taylor \& Bruenn 2009; Taylor et al. 2011; Taylor et al. 2010) or via RNA silencing.

\section{CONCLUSIONS}

The existence of mitovirus NERVEs in plant mitochondria (Marienfeld et al. 1997) is remarkable for several reasons. First, the mitovirus NERVEs are clearly derived from fungal mitoviruses rather than from some hypothetical ancestral virus native to plant mitochondria. Given that the only encapsidated viruses to which the mitovirus are related are the leviviruses of 
188 bacteria, it is tempting to postulate that the mitoviruses of fungi are remnants of the original capture of bacteria by eukaryotes (the ancestors of mitochondria) and therefore predate the divergence of plants and animals (Koonin \& Dolja 2014). However, there are currently no known plant (or animal) Narnaviridae, and the monophyletic nature of the plant mitovirus NERVEs and their presence in only a subset of plants does not support this model for the origin of mitoviruses. Rather it implies transfer from fungal mitochondria to plant mitochondria, as has been suggested previously (Marienfeld et al. 1997; Xu et al. 2015). Given the intimate association of plant pathogenic fungi with their hosts, this seems possible, especially given the known horizontal transfer of plant mitochondria within the plant kingdom (Leister \& Kleine 2011). Plant endophytic fungi are an even more likely origin (Bao \& Roossinck 2013). A similar origin for the plant endornaviruses by horizontal transfer of some segments from fungal endornaviruses has been proposed (Koonin \& Dolja 2014; Rastgou et al. 2009). Second, like all NERVEs, integration into DNA genomes requires reverse transcription. Where it is possible to trace the origin of a NERVE integration event, integration is the result of promiscuous substrate switching by a transposon reverse transcriptase (Ballinger et al. 2012). Presumably this is the case for the mitovirus NERVEs. Third, the plant mitochondrial genetic code is the standard code, while the fungal mitochondrial genetic code uses UGA as a tryptophan codon, so the mitovirus NERVEs all must use the standard code. This implies the switching of all UGA codons to UGG either prior to the integration event or afterwards (Shackelton \& Holmes 2008). The switch could easily have happened prior to integration, so that a fungal mitovirus adapted to growth in plant mitochondria and the plant responded by integrating a copy of its RdRP in such a way as to interfere with viral replication. Fungal viruses with larger genomes than that of the mitoviruses are known to successfully adapt to hosts with alternate genetic codes (Taylor et al. 2013). In addition, the Saccharomyces cerevisiae narnaviruses have escaped from mitochondria, replicate in the cytoplasm, and consequently use the standard genetic code (Koonin \& Dolja 2014).

There are three methods by which we might corroborate a single integration of a mitovirus cDNA into a plant mitochondrial genome: correspondence of a mitovirus NERVE phylogeny with that of plant mitochondria; synteny of regions around mitovirus NERVE integration sites among plant mitochondrial genomes; or monophyly of mitovirus NERVEs. Unfortunately, a reliable mitovirus NERVE phylogeny is impossible to construct with current data, since the conserved regions of the RdRP do not vary significantly among the mitovirus NERVEs and the regions between them show very little sequence conservation. A much larger collection of mitovirus NERVEs would be required to construct a phylogeny with any confidence. In addition, there is not enough synteny among plant mitochondrial genomes to detect conservation around NERVE integration sites. It is possible that multiple integration events took place, but the monophyletic nature of the mitovirus NERVEs and their preservation of the functional motifs of the RdRP argues for a single integration event. If there was a single integration event, it had to take place prior to the origin of vascular plants in the early Silurian (Steemans et al. 2009), providing the earliest evidence for viruses of any kind, about 400 million years ago, older than the estimate of 310 million years ago for insect DNA viruses (Theze et al. 2011).

\section{ACKNOWLEDGEMENTS}

We thank Charlotte Lindqvist, Jeffrey Boore, Matt Ballinger, and Riva Bruenn for comments on the manuscript. 


\section{ADDITIONAL INFORMATION AND DECLARATIONS}

Funding

Material support was provided by the University at Buffalo. The funders had no role in study

design, data collection and analysis, decision to publish, or preparation of the manuscript.

Competing interests

J. Bruenn is an editor for PeerJ.

Author contributions

Jeremy A. Bruenn conceived and designed the experiments, performed the experiments, analyzed the data, contributed reagents/materials/analysis tools, wrote the paper.

Pradeep Yerrmasetty and Ben Warner conceived and designed experiments, performed experiments, and contributed reagents/materials/analysis tools.

Supplemental information

Supplemental information for this article can be found online at

\section{REFERENCES}

Abascal F, Zardoya R, and Posada D. 2005. ProtTest: selection of best-fit models of protein evolution. Bioinformatics 21:2104-2105.

Al Rwahnih M, Daubert S, Urbez-Torres JR, Cordero F, and Rowhani A. 2011. Deep sequencing evidence from single grapevine plants reveals a virome dominated by mycoviruses. Arch Virol 156:397-403.

Altschul SF, Gish W, Miller W, and Myers EW. 1990. Basic local alignment search tool. J Molec Biol 215:403-410.

Altschul SF, Madden TL, Schaffer AA, Zhang J, Zhang Z, Miller W, and Lipman DJ. 1997. Gapped BLAST and PSI-BLAST: a new generation of protein database search programs. Nucleic Acids Res 25:3389-3402.

Anisimova M, and Gascuel 0. 2006. Approximate likelihood-ratio test for branches: A fast, accurate, and powerful alternative. Syst Biol 55:539-552.

Ballinger MJ, Bruenn JA, Kotov AA, and Taylor DJ. 2013. Selectively maintained paleoviruses in Holarctic water fleas reveal an ancient origin for phleboviruses. Virology 446:276-282.

Ballinger MJ, Bruenn JA, and Taylor DJ. 2012. Phylogeny, integration and expression of sigma virus-like genes in Drosophila. Molecular phylogenetics and evolution 65:251-258.

Bao X, and Roossinck MJ. 2013. Multiplexed interactions: viruses of endophytic fungi. Adv Virus Res 86:37-58.

Bruenn JA. 2003. A structural and primary sequence comparison of the viral RNA-dependent RNA polymerases. Nucleic Acids Res 31:1821-1829.

Cai G, Myers K, Fry WE, and Hillman BI. 2012. A member of the virus family Narnaviridae from the plant pathogenic oomycete Phytophthora infestans. Arch Virol 157:165-169. 
Chang JM, Di Tommaso P, and Notredame C. 2014. TCS: a new multiple sequence alignment reliability measure to estimate alignment accuracy and improve phylogenetic tree reconstruction. Mol Biol Evol 31:1625-1637.

Cook S, Chung BY, Bass D, Moureau G, Tang S, McAlister E, Culverwell CL, Glucksman E, Wang H, Brown TD, Gould EA, Harbach RE, de Lamballerie X, and Firth AE. 2013. Novel virus discovery and genome reconstruction from field RNA samples reveals highly divergent viruses in dipteran hosts. PLoS One 8:e80720.

Crochu S, Cook S, Attoui H, Charrel RN, De Chesse R, Belhouchet M, Lemasson JJ, de Micco P, and de Lamballerie X. 2004. Sequences of flavivirus-related RNA viruses persist in DNA form integrated in the genome of Aedes spp. mosquitoes. J Gen Virol 85:1971-1980.

Cui J, and Holmes EC. 2012. Endogenous RNA viruses of plants in insect genomes. Virology 427:77-79.

Davis CC, Xi Z, and Mathews S. 2014. Plastid phylogenomics and green plant phylogeny: almost full circle but not quite there. BMC Biol 12:11.

Esteban LM, Fujimura T, Garcia-Cuellar M, and Esteban R. 1994. Association of yeast viral $23 \mathrm{~S}$ RNA with its putative RNA-dependent RNA polymerase.J Biol Chem 269:29771-29777.

Esteban LM, Rodriguez-Cousino N, and Esteban R. 1992. T double-stranded RNA (dsRNA) sequence reveals that $\mathrm{T}$ and $\mathrm{W}$ dsRNAs form a new RNA family in Saccharomyces cerevisiae. Identification of $23 \mathrm{~S}$ RNA as the single-stranded form of T dsRNA.J Biol Chem 267:10874-10881.

Fukuhara T, Koga R, Aoki N, Yuki C, Yamamoto N, Oyama N, Udagawa T, Horiuchi H, Miyazaki S, Higashi Y, Takeshita M, Ikeda K, Arakawa M, Matsumoto N, and Moriyama H. 2006. The wide distribution of endornaviruses, large double-stranded RNA replicons with plasmidlike properties. Arch Virol 151:995-1002.

Garcia-Cuellar MP, Esteban LM, Fujimura T, Rodriguez-Cousino N, and Esteban R. 1995. Yeast viral $20 \mathrm{~S}$ RNA is associated with its cognate RNA-dependent RNA polymerase. J Biol Chem 270:20084-20089.

Garcia-Cuellar MP, Esteban R, and Fujimura T. 1997. RNA-dependent RNA polymerase activity associated with the yeast viral p91/20S RNA ribonucleoprotein complex. Rna 3:27-36.

Gibbs MJ, Koga R, Moriyama H, Pfeiffer P, and Fukuhara T. 2000. Phylogenetic analysis of some large double-stranded RNA replicons from plants suggests they evolved from a defective single-stranded RNA virus. J Gen Virol 81:227-233.

Hillman BI, and Cai G. 2013. The family narnaviridae: simplest of RNA viruses. Adv Virus Res 86:149-176.

Hong Y, Cole TE, Brasier CM, and Buck KW. 1998. Evolutionary relationships among putative RNA-dependent RNA polymerases encoded by a mitochondrial virus-like RNA in the Dutch elm disease fungus, Ophiostoma novo-ulmi, by other viruses and virus-like RNAs and by the Arabidopsis mitochondrial genome. Virology 246:158-169.

Horie M, Honda T, Suzuki Y, Kobayashi Y, Daito T, Oshida T, Ikuta K, Jern P, Gojobori T, Coffin JM, and Tomonaga K. 2010. Endogenous non-retroviral RNA virus elements in mammalian genomes. Nature 463:84-87.

Katoh K, Misawa K, Kuma K, and Miyata T. 2002. MAFFT: a novel method for rapid multiple sequence alignment based on fast Fourier transform. Nucleic Acids Res 30:3059-3066.

Katzourakis A, and Gifford RJ. 2010. Endogenous viral elements in animal genomes. PLoS Genet 6:e1001191. 
Kearse M, Moir R, Wilson A, Stones-Havas S, Cheung M, Sturrock S, Buxton S, Cooper A, Markowitz S, Duran C, Thierer T, Ashton B, Meintjes P, and Drummond A. 2012. Geneious Basic: an integrated and extendable desktop software platform for the organization and analysis of sequence data. Bioinformatics 28:1647-1649.

Koonin EV, Choi GH, Nuss DL, Shapira R, and Carrington JC. 1991. Evidence for common ancestry of a chestnut blight hypovirulence-associated double-stranded RNA and a group of positive-strand RNA plant viruses. Proc Natl Acad Sci USA 88:10647-10651.

Koonin EV, and Dolja VV. 2013. A virocentric perspective on the evolution of life. Curr Opin Virol 3:546-557.

Koonin EV, and Dolja VV. 2014. Virus world as an evolutionary network of viruses and capsidless selfish elements. Microbiol Mol Biol Rev 78:278-303.

Leister D, and Kleine T. 2011. Role of intercompartmental DNA transfer in producing genetic diversity. Int Rev Cell Mol Biol 291:73-114.

Lyons E, and Freeling M. 2008. How to usefully compare homologous plant genes and chromosomes as DNA sequences. Plant J 53:661-673.

Marienfeld JR, Unseld M, Brandt P, and Brennicke A. 1997. Viral nucleic acid sequence transfer between fungi and plants. Trends Genet 13:260-261.

Osaki H, Nakamura H, Nomura K, Matsumoto N, and Yoshida K. 2005. Nucleotide sequence of a mitochondrial RNA virus from the plant pathogenic fungus, Helicobasidium mompa Tanaka. Virus Res 107:39-46.

Palmer JD, and Herbon LA. 1988. Plant mitochondrial DNA evolves rapidly in structure, but slowly in sequence. J Mol Evol 28:87-97.

Rastgou M, Habibi MK, Izadpanah K, Masenga V, Milne RG, Wolf YI, Koonin EV, and Turina M. 2009. Molecular characterization of the plant virus genus Ourmiavirus and evidence of inter-kingdom reassortment of viral genome segments as its possible route of origin. J Gen Virol 90:2525-2535.

Raven PH. 1970. A multiple origin for plastids and mitochondria. Science 169:641-646.

Rodriguez-Cousino N, Esteban LM, and Esteban R. 1991. Molecular cloning and characterization of W double-stranded RNA, a linear molecule present in Saccharomyces cerevisiae. Identification of its single-stranded RNA form as 20 S RNA. J Biol Chem 266:12772-12778. Rodriguez-Cousino N, and Esteban R. 1992. Both yeast W double-stranded RNA and its singlestranded form 20S RNA are linear. Nucleic Acids Res 20:2761-2766.

Roossinck MJ. 2010. Lifestyles of plant viruses. Philos Trans R Soc Lond B Biol Sci 365:1899-1905. Shackelton LA, and Holmes EC. 2008. The role of alternative genetic codes in viral evolution and emergence. J Theor Biol 254:128-134.

Steemans P, Herisse AL, Melvin J, Miller MA, Paris F, Verniers J, and Wellman CH. 2009. Origin and radiation of the earliest vascular land plants. Science 324:353.

Taylor DJ, Ballinger MJ, Bowman SM, and Bruenn JA. 2013. Virus-host co-evolution under a modified nuclear genetic code. PeerJ 1:e50.

Taylor DJ, and Bruenn J. 2009. The evolution of novel fungal genes from non-retroviral RNA viruses. BMC Biol 7:88.

Taylor DJ, Dittmar K, Ballinger MJ, and Bruenn JA. 2011. Evolutionary maintenance of filoviruslike genes in bat genomes. BMC Evol Biol 11:336.

Taylor DJ, Leach RW, and Bruenn J. 2010. Filoviruses are ancient and integrated into mammalian genomes. BMC Evol Biol 10:193. 
369 Theze J, Bezier A, Periquet G, Drezen JM, and Herniou EA. 2011. Paleozoic origin of insect large dsDNA viruses. Proc Natl Acad Sci U S A 108:15931-15935.

Xi Z, Wang Y, Bradley RK, Sugumaran M, Marx CJ, Rest JS, and Davis CC. 2013. Massive mitochondrial gene transfer in a parasitic flowering plant clade. PLoS Genet 9:e1003265. mitochondrial gene confers hypovirulence on the phytopathogenic fungus Sclerotinia sclerotiorum. Virus Research 197:127-136. 


\section{1}

Consensus sequence of narnavirus NERVES

Consensus sequence of 175 mitovirus NERVEs and 29 mitovirus RdRPs and similarity plot along the sequence. FABCDE indicate the conserved motifs of RdRPs. Alignment was generated by MAFFT as described in Materials and Methods.

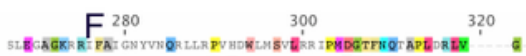

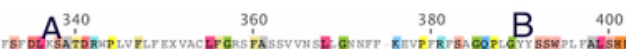

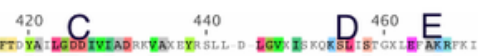

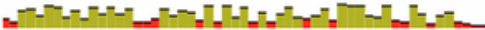


2

Synteny between mitochondrial and chromosomal genomes including regions with mitovirus NERVES

Synteny between mitochondrial and chromosomal genomes including regions with mitovirus

NERVEs (indicated by NN) for three plant species. The Arabidopsis mitovirus NERVE used in the RTPCR experiment of Fig. 5 is indicated in the top two panels. Synteny diagrams were generated by CoGe, as described in Materials and Methods

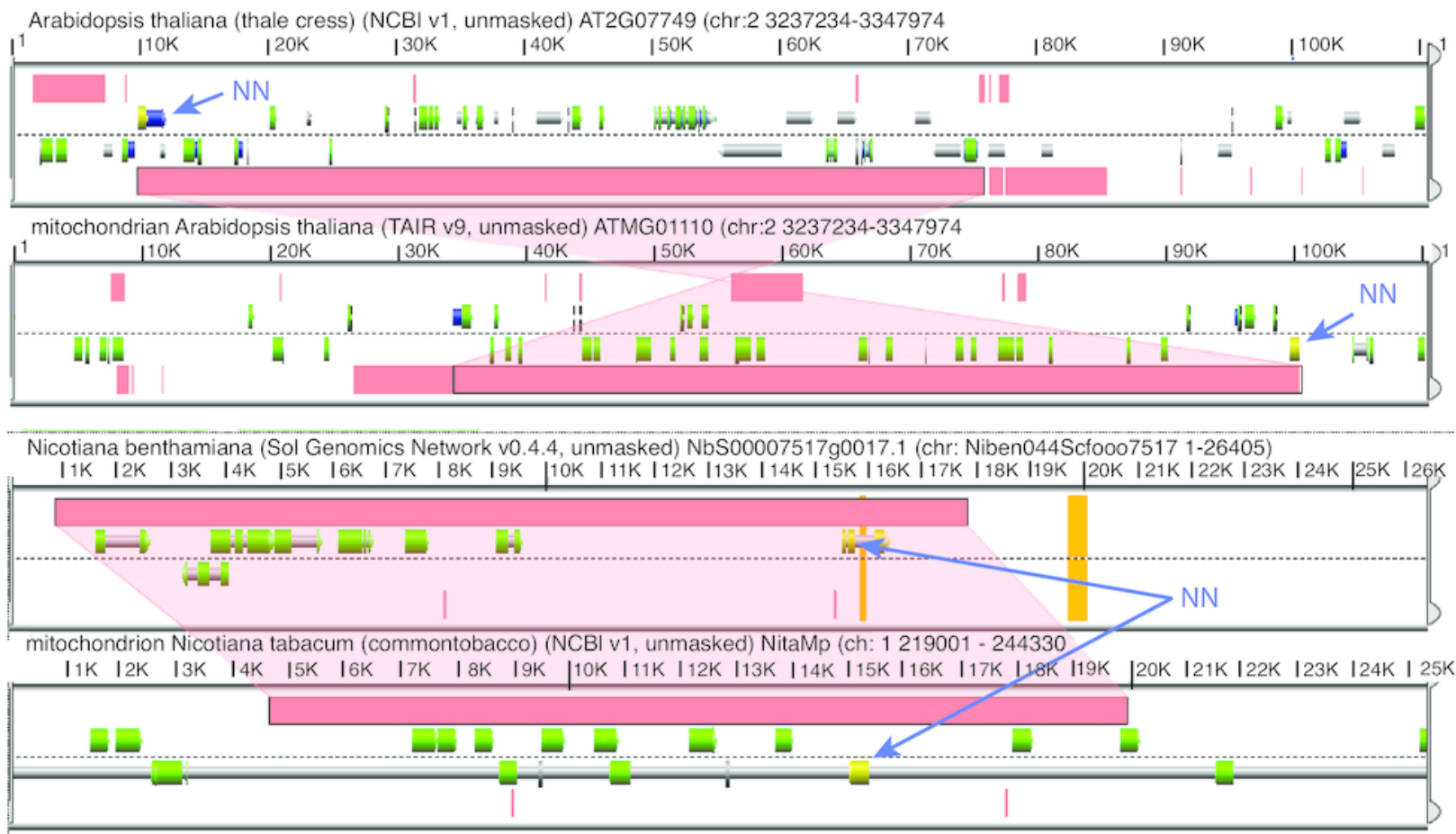

Vitis vinifera (NCBI v2, unmasked) LOG100258057 (ch: 108023461 - 8044250)

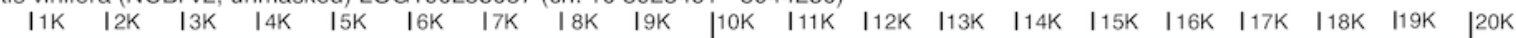

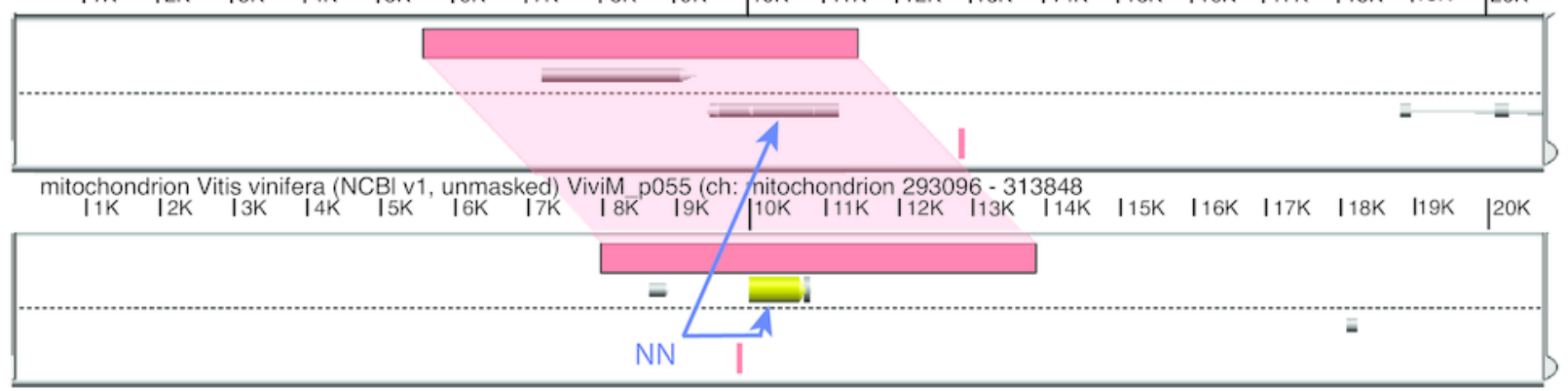


3

Abbreviated cladogram of the Viridiplantae according to Davis (Davis et al. 2014) .

Abbreviated cladogram of the Viridiplantae according to Davis (Davis et al. 2014). The plant groups in which mitovirus NERVEs have been demonstrated are indicated by black dots. All of the groups of vascular plants without demonstrated mitovirus NERVEs (e.g. ferns, Pinaceae, etc.) have no sequenced mitochondrial genomes

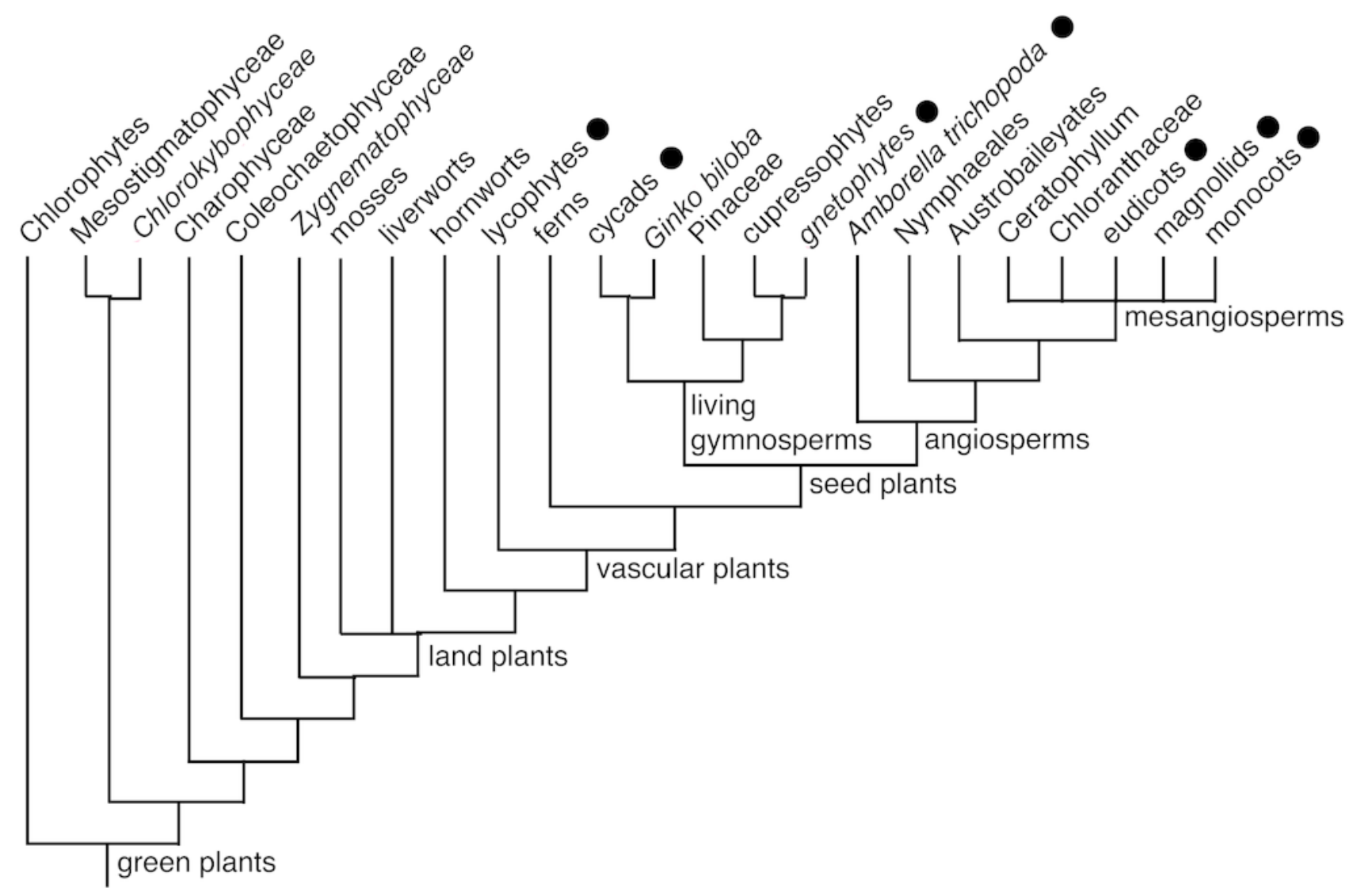




\section{4}

Abbreviated phylogeny of the mitovirus NERVEs and the known narnaviruses

Abbreviated phylogeny of the mitovirus NERVEs and the known narnaviruses. The sequences of 61 plant mitochondrial mitovirus NERVEs of 100 amino acids or longer in length were aligned with the 31 known narnavirus RdRPs and two representative levivirus RdRPs and a phylogram rooted by the levivirus sequences generated as described in Materials and Methods. aLRT support values are shown. Several mitoviruses listed by name are ambiguous because more than one mitovirus is present. These are Ophistioma mitovirus 1c (AGT55876), Sclerotinia sclerotiorum mitovirus 7 (AHE13866) and Sclerotinia homeocarpa mitovirus (AAO21337). 


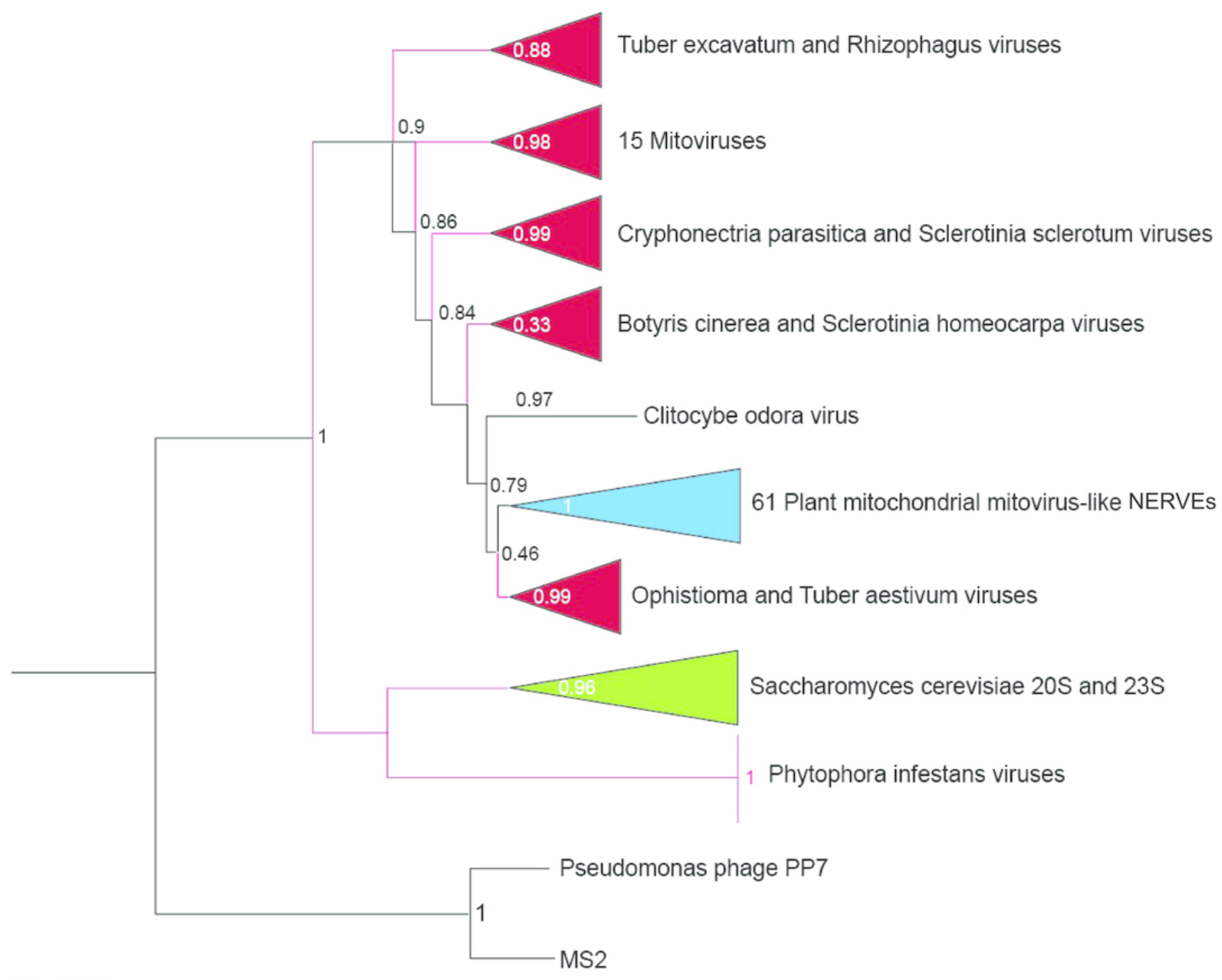




\section{5}

RTPCR of Arabidopsis thaliana total nucleic acids

Reactions before (DNA) or after (RNA) DNase treatment were tested for the nuclear or mitochondrial mitovirus NERVE or for the control (rbcL) sequence and analyzed on a $1.4 \%$ agarose gel as described in Materials and Methods. The first lane has DNA size markers, of which two are shown, one of $500 \mathrm{bp}$ and one of $250 \mathrm{bp}$.

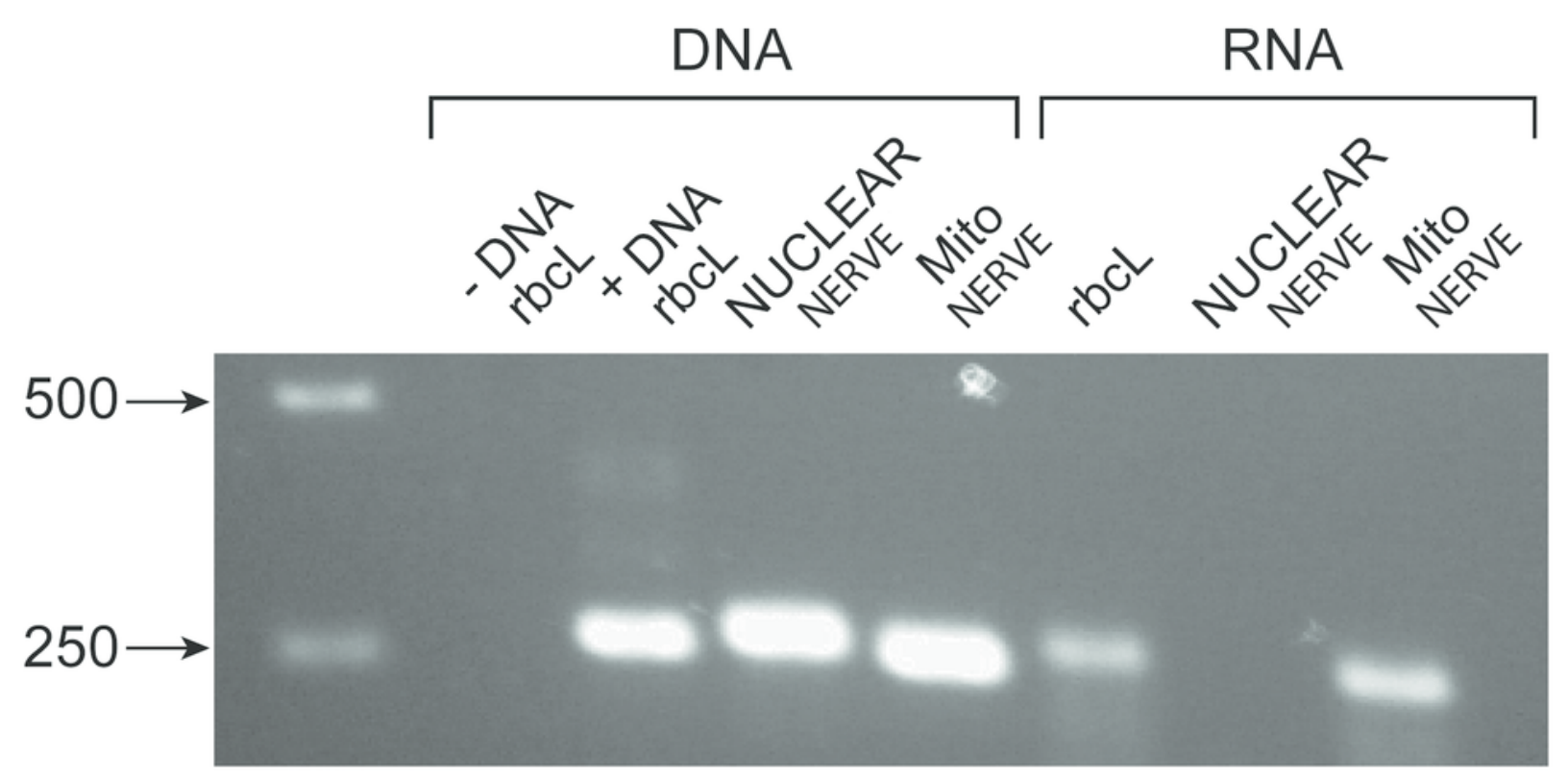

